\title{
Analysis of the associations between missense substitutions in the human MT-ATP6 gene
}

\author{
Maria Golubenko \\ Laboratory of population genetics \\ Research Institute of Medical Genetics, Tomsk NRMC \\ Tomsk, Russia \\ maria.golubenko@medgenetics.ru
}

\author{
Alexey Zarubin \\ Laboratory of evolutionary genetics \\ Research Institute of Medical Genetics, Tomsk NRMC \\ Tomsk, Russia \\ aleksei.zarubin@medgenetics.ru
}

\begin{abstract}
Mitochondrial DNA encodes 13 subunits of mitochondrial respiratory chain complexes. There is high mtDNA variability in human populations, and substantial part of it is regional specific. It is hypothesized that mtDNA common variants can be adaptive and have some effect on the mitochondrial function. MT-ATP6 gene is particularly variable in human populations in regard of missense polymorphisms. We have analyzed MT-ATP6 missense substitutions in the mtDNA haplogroups which are common for Northern Asia (Siberia). Representatives of mitochondrial haplogroups A, D4, C4, C5, Z, and $L$ (the latter as an example of a "Southern" haplogroup) were selected from the GenBank collection of whole human mtDNA sequences, and amino acid sequences encoded by ATP6 were analyzed. We show that amino acid variability in the ATP6 depends on the substitutions in the roots of these haplogroups. In particular, Ala59Thr in combination with His90Tyr substitution (haplogroup A) are associated with a number of polymorphic amino acids in the first 60 positions of ATP6, whereas ancestral Ala59 variant (haplogroup D), either alone or in the combination with Ala20Thr substitution (haplogroups $\mathrm{C4}, \mathrm{C5}$, and $\mathrm{Z}$ ), seems to have "suppressing" effect on the variability in this region. Comparison of the estimated "coupling values" (which reflect associations between amino acids in the protein) between each of these three positions and all amino acid positions in the 1-59 region has revealed that mean coupling values were comparatively higher for the position 20, lower for the position 59, and the lowest for the position 90 ( $p=0.0002)$. In contrast, for the missense substitutions which actually have occurred in these haplogroups, the mean coupling values were close to 0 at all three positions. Our results demonstrate possible epistatic effect of the haplogroup-defining missense variants in the mtDNA encoded protein.
\end{abstract}

Keywords - mitochondrial DNA, molecular phylogeny, adaptation

\section{Introduction}

Mitochondrial DNA (mtDNA) is widely used in the population and evolutionary genetics, due to its uniparental inheritance and high mutation rate. In human populations, mtDNA variation has been extensively studied, and detailed intraspecific phylogeny was reconstructed and is available online [1]. All mtDNA haplotypes can be classified as belonging to different haplogroups which share common nucleotide substitutions. Along with silent and non-coding substitutions, these haplogroup-specific polymorphisms include amino acid changes in mtDNA-encoded subunits of mitochondrial respiratory chain complexes, as well as nucleotide substitutions in the mitochondrial rRNA and tRNA genes. Additional polymorphisms occur at the terminal branches of the tree. MtDNA variation has substantial geographic differentiation, so that main branches of the tree are continent-specific. It was proposed that not the genetic drift alone, but natural selection as well could contribute into the global distribution of the mtDNA variation [2], and that some mtDNA haplogroups might have slightly increased or decreased respiratory chain efficiency, contributing to the adaptation to climate and/or food sources [3].
It is possible that the sequential accumulation of mutations along the mtDNA molecule in the course of human microevolution is not random, because of interactive and cumulative effects of several mutations, but such effects are difficult to estimate. The aim of our study was to search for possible patterns of amino acid replacements in the human mtDNA phylogeny, using the MT-ATP6 gene as an example.

\section{Methods}

For the analysis of the intraspecific phylogeny of human mtDNA, a data set was generated which contains all complete human mitochondrial genome sequences (48935 sequences) available in the NCBI Nucleotide database. These sequences were aligned to the rCRS reference sequence using bwa bwasw, and then a list of substitutions was obtained from the sequences. The detected substitutions were used for verification of the haplogroup using HaploGrep [4]. Sequences with low confidence of haplogroup affiliation (less than 0.9) were excluded from the analysis. Then, several haplogroups were chosen which had experienced expansion in Northeastern Eurasia (A, D4, C4, C5, Z), as well as one "Southern" haplogroup L3 which had expansion in Africa. For these haplogroups, amino acid sequences for ATP6 were translated from the DNA sequence data. List of amino acid replacements and their conservation indices and MutPred values were obtained using MtPhyl program [5]. In addition, estimates of mtDNA selection scores [6] and coupling values for amino acid pairs [7] were analyzed. Statistical comparisons were performed in the Statistica 8.0 package (StatSoft Inc.).

\section{Results}

ATP6 protein consists of 226 amino acids. The number of polymorphic amino acid positions in the studied data set was 51 . Almost all sequences in all haplogroups have the "Thr112Ala" substitution (A8860G), where Alanine is in fact ancestral state, because the reference sequence (rCRS) belongs to the haplogroup H2a2a which has Ala112Thr substitution. Also, all haplogroups except of the haplogroup A have Thr59Ala substitution $(\mathrm{A} 8701 \mathrm{G})$, again with Alanine being the ancestral allele (the Ala59Thr change had occurred in the macrohaplogroup $\mathrm{N}$, from which macrogaplogroup $\mathrm{R}$ originated, including $\mathrm{H} 2 \mathrm{a} 2$ with the rCRS). Haplogroups $\mathrm{C} 4$, $\mathrm{C} 5, \mathrm{Z}$, D4 belong to the macrogaplogroup $\mathrm{M}$, which originated directly from L3 (the root for non-African haplogroups), therefore they also have this difference from the reference sequence. Haplogroup A belongs to N, so it has threonine in this position. However, almost all haplotypes of haplogroup A have a different substitution in the same part of the protein sequence, His90Tyr (C8794T). Representatives of the M branches (C4, C5, Z - all belonging to the M8 cluster) have another replacement in the Nterminus of ATP6 - Ala20Thr (G8584A).

The total number of polymorphic positions in each haplogroup should depend on its age and prevalence: the 
probability of amino acid change is higher in the older and more abundant haplogroups, regardless of their adaptive significance. In the analyzed data set, haplogroup D4 was the most variable, with the coalescence time of about 26 thousand years, [8] and the number of the analyzed sequences (1323) was the largest. Analysis of the haplogroup D4 revealed 24 polymorphic amino acids. The coalescence time of haplotypes of haplogroup L3b was 12-15 thousands years ago [9], and for this cluster, 11 polymorphic positions were observed. The least variability in our dataset was demonstrated for the haplogroups $\mathrm{Z}$ and $\mathrm{C} 5$, with the coalescence time estimated as 16.5 and 20 thousand years ago [8], and the number of analyzed haplotypes is 175 and 120 , respectively. Only one polymorphic amino acid was detected in haplogroup C5 (in addition to Ala20Thr), and in haplogroup $\mathrm{Z}$ there were 7 polymorphic amino acids. From the other side, the coalescence time for both haplogroups A $(n=697)$ and $\mathrm{C} 4(\mathrm{n}=570)$, was estimated at about 21 thousand years [8, 10], but there was significant difference in amino acid ATP6 variability: 22 polymorphic amino acids (in addition to His90Tyr) in the haplogroup A and only 4 (in addition to Ala20Thr) in the haplogroup C4. So, there is decreased protein variation in the haplogroup $\mathrm{C} 4$, versus the increased variability in the haplogroup A, despite similar age and prevalence.

When considering the pattern of the ATP6 amino acid sequence polymorphism in the studied haplogroups, it can be noted that the presence of threonine at position 59 (haplogroup A) compared with alanine (the remaining halpogroups) is associated with an increase in variability in the N-terminal part of the protein: in the region from position 1 to 59 , there are 11 polymorphic amino acids in haplogroup A, compared to 6 in haplogroup D and 3 in haplogroup C4. It should be noted, however, that all $\mathrm{C} 4$ representatives in this area have a replacement Ala20Thr (occurred at the root of M8 haplogroup which encompasses the haplogroup C). The explanation for the observed picture may be the adaptive effect of the Ala59Thr substitution, which weakens the pressure of negative selection. Another explanation could be interaction of this amino acid with the $\mathrm{N}$-terminal part of the protein, i.e. the epistatic effect of substitution in this position. An indirect assessment of epistatic effects can be given by the method of analysis of interactions published in [7]. In that study, based on a comparative analysis, data on the "coupling values" between amino acids at various positions for several thousands known proteins, including the sixth subunit of ATP synthase, were calculated, and the results have been made publicly available. It was assumed that in the absence of interaction between amino acids, the average value of this index should be close to zero. Using these data, we selected and compared the calculated estimates of the coupling values of positions 20,59, and 90 (where haplogroup-specific amino acid substitutions occurred in the studied haplogroups) with all amino acids in the N-terminal part of the protein where we found haplogroup-specific variability (i.e. amino acids 1658). The analysis of variance revealed statistically significant differences in the average coupling values between the three positions $(p=0,0002)$. Maximum average coupling value was estimated for the amino acid at the position 20 , the intermediate value for the position 59, and the minimum for the position 90 (Fig.1). In contrast, mean coupling values for the positions 20, 59, 90 and actually occurred polymorphic amino acids did not differ from 0 . Thus, the results suggest possible effect of the amino acid changes at the root of a haplogroup on the further protein variability. However, this question requires further research - in particular, a more complete analysis of other mtDNA haplogroups would allow to determine whether the detected association of threonine 59 with higher variability in the N-terminal part of the protein is preserved in the branches of $\mathrm{N}$ and $\mathrm{R}$ macrohaplogroups. In general, the high number of polymorphic positions in the ATP6 gene in the "northern" haplogroups $\mathrm{D}$ and $\mathrm{A}$ is consistent with the previously described phenomenon of high amino acid variability of ATP6 in the northern latitudes $[2,11]$. The relatively low variability in the haplogroups $\mathrm{C}$ and $\mathrm{Z}$ can be associated with the presumable effect of the Ala20Thr substitution.

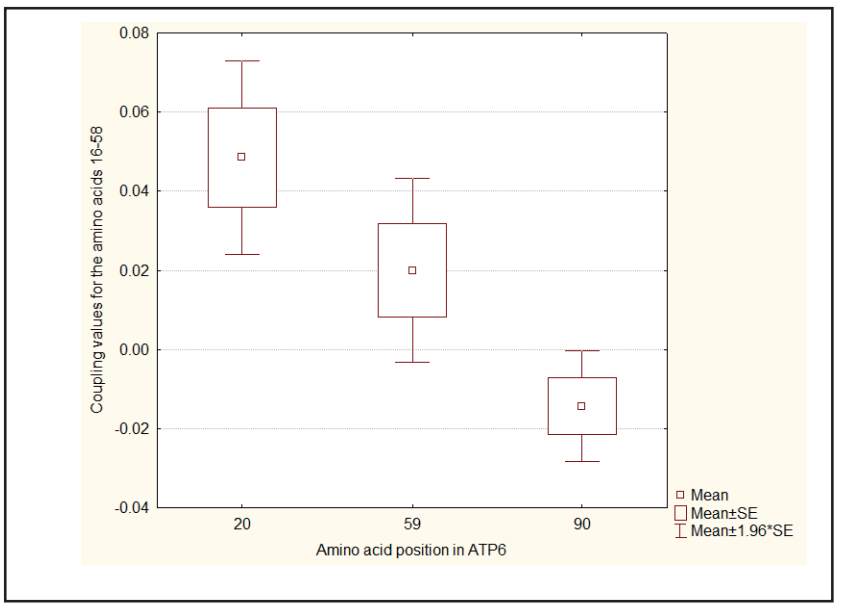

Fig. 1. Mean coupling values for the amino acids 20, 59, and 90 with the amino acids $16-58$ in ATP6.

\section{References}

[1] M. van Oven and M. Kayser, "Updated comprehensive phylogenetic tree of global human mitochondrial DNA variation, Hum. Mutat., vol. 30, pp E386-E394, 2009.

[2] E. Ruiz-Pesini, D. Mishmar, M. Brandon, V. Procaccio, D.C. Wallace, "Effects of purifying and adaptive selection on regional variation in human mtDNA," Science, vol. 303, pp. 223-226, January 2004.

[3] D. C. Walace, "Mitochondrial DNA Variation in Human Radiation and Disease", Cell, vol. 163, pp. 33-38, 2015.

[4] H. Weissensteiner, D. Pacher, A. Kloss-Brandstätter, L. Forer, G. Specht, H. J. Bandelt, F. Kronenberg, A. Salas, S. Schönherr, "HaploGrep 2: mitochondrial haplogroup classification in the era of high-throughput sequencing," Nucleic Acids Res., vol. 44 (W1), pp. W58-63, July 2016.

[5] N. Eltsov and N. Volodko, "New method and new tool for human mtDNA phylogeny reconstruction," Europ. J. Hum. Genet., vol 17, suppl. 1, p. 298, May 2009 [Abstracts of the European Human Genetic Conference, Vienna, P 11.079

[6] L. Pereira, P. Soares, P. Radivojac, B. Li, D. C. Samuels, "Comparing phylogeny and the predicted pathogenicity of protein variations reveals equal purifying selection across the global human mtDNA diversity," Am. J. Hum. Genet., vol. 88, pp. 433-439, April 2011.

[7] T.A. Hopf, J.B. Ingraham, F.J. Poelwijk, C.P. Schärfe, M. Springer, C. Sander, D.C. Marks, "Mutation effects predicted from sequence covariation," Nat. Biotechnol., vol. 35, pp. 128-135, Februalry 2017.

[8] M. Derenko, B. Malyarchuk, T. Grzybowski, G. Denisova, U. Rogalla, M. Perkova, I. Dambueva, I. Zakharov, "Origin and post-glacial dispersal of mitochondrial DNA haplogroups C and D in northern Asia," PLoS One, vol. 5, p. e15214, December 2010.

[9] P. Soares, F. Alshamali, J. B. Pereira, V. Fernandes, N. M. Silva, C. Afonso, M.D. Costa, E. Musilová, V. Macaulay, M. B. Richards, V. Cerny, L. Pereira, "The Expansion of mtDNA Haplogroup L3 within and out of Africa," Mol Biol Evol., vol. 29, pp. 915-927, March 2012.

[10] M. Derenko M, Malyarchuk B, Grzybowski T, Denisova G, Dambueva I, Perkova M, Dorzhu C, Luzina F, Lee HK, Vanecek T, Villems R, Zakharov I. Phylogeographic analysis of mitochondrial DNA in northern Asian populations. Am J Hum Genet. 2007 Nov;81(5):1025-41.

[11] M. Ingman and U. Gyllensten, Rate variation between mitochondrial domains and adaptive evolution in humans. Hum Mol Genet. 2007 Oct 1;16(19):2281-7. 